\section{Validação de Questionários de \\ Freqüência Alimentar - QFA: \\ considerações metodológicas}

\author{
Validation of Food Frequency \\ Questionnaires - FFQ: \\ methodological considerations
}

\section{Betzabeth Slater}

Departamento de Nutrição

Faculdade de Saúde Pública

Universidade de São Paulo

Av. Dr. Arnaldo, 715 - Cerqueira Cesar

01246-904 - São Paulo - SP

betzy_slater@hotmail.com

\section{Sonia Tucunduva Philippi}

Departamento de Nutrição

Faculdade de Saúde Pública

Universidade de São Paulo

Dirce M. L. Marchioni

Universidade Metodista de São Paulo

\section{Regina Mara Fisberg}

Departamento de Nutrição

Faculdade de Saúde Pública

Universidade de São Paulo

\section{Resumo}

O Questionário de Freqüência Alimentar (QFA) tornou-se o método dominante nos estudos epidemiológicos para avaliação do consumo dietético, em especial para avaliar a relação da dieta com a ocorrência de doenças crônicas não transmissíveis. O QFA é um instrumento que tem como objetivo a avaliação da dieta habitual de grupos populacionais e apresenta como vantagens a rapidez na aplicação e a eficiência na prática epidemiológica para identificar o consumo habitual de alimentos, além do baixo custo. Comparado a outros instrumentos, substitui a medição da ingestão alimentar de um ou vários dias pela informação global da ingestão de um período amplo de tempo. Constitui ferramenta útil nos estudos sempre que seu desenvolvimento tenha sido precedido por procedimentos metodológicos cuidadosamente planejados que garantirão confiabilidade e precisão dos dados. Para tanto, são necessários estudos de validação que permitam estimar os erros de medição próprios do método. Sem o reconhecimento destes erros, a relação dieta-doença poderá estar prejudicada. Este artigo pretende abordar de forma sistemática considerações metodológicas para a realização de estudos de validação de QFA.

Palavras-chave: Questionários de freqüência alimentar. Estudo de validação. Dieta habitual. 


\section{Abstract}

The Food Frequency Questionnaire (FFQ) has become the dominant method for assessing dietary intakes in epidemiological studies, especially to evaluate the relationship of diet with the occurrence of non-transmissible chronic diseases. The FFQ is a tool designed to evaluate the usual diet of a population and has the advantages of being easy and quick to apply and efficient in the epidemiological practice of identifying usual food consumption, in addition to its low cost. Compared to other tools, it replaces the measurement of one or several days of food intake with overall information on the intake over a more extended period of time. It becomes a useful tool in studies, when its use is preceded by carefully planned methodological procedures, to ensure the reliability and accuracy of the data. For such purpose, validation studies are required to estimate measuring errors related to the method. If these errors are not known, the true effect of a diet-disease relationship may be jeopardized. This article is a systematic approach to methodological considerations for FFQ validation studies.

Keywords: Food Frequency Questionnaire. Validation study. Usual Intake.

\section{Introdução}

O questionário de freqüência alimentar (QFA) é considerado como o mais prático e informativo método de avaliação da ingestão dietética e fundamentalmente importante em estudos epidemiológicos que relacionam a dieta com a ocorrência de doenças crônicas $^{1-3}$.

Desde a década de 60, o método de freqüência alimentar tem sido considerado uma ferramenta simples, econômica e capaz de distinguir os diferentes padrões de consumo entre os indivíduos ${ }^{4}$.

Conceitualmente, o método prevê a medição da exposição e sua relação com o tempo, de maneira a refletir características de como começa, quando termina e qual a sua distribuição no período de interesse ${ }^{5,6}$.

Um dos objetivos implícitos do QFA é conhecer o consumo habitual de alimentos por um grupo populacional e, neste sentido, a estrutura do instrumento contempla o registro da freqüência de consumo de alimentos em unidades de tempo.

Entre as vantagens que o QFA oferece está a rapidez da aplicação e a eficiência na prática epidemiológica para identificar o consumo habitual de alimentos. O QFA, comparado a outros métodos, substitui a medição da ingestão alimentar de um ou vários dias pela informação global da ingestão de um período amplo de tempo ${ }^{7}$.

O QFA pode ser uma ferramenta útil nos estudos epidemiológicos sempre e quando o desenvolvimento do método tenha sido precedido por procedimentos metodológicos cuidadosamente planejados, que posteriormente garantirão maior confiabilidade e precisão dos dados. Os estudos de validação, portanto, estimarão os erros de medição próprios do método, que são vistos como a principal fonte de viés nos estudos epidemiológicos.

Este trabalho aborda de forma sistemática o delineamento e os procedimentos metodológicos necessários para a realização de estudos de validação de Questionários de Freqüência Alimentar - QFA. 


\section{Desenvolvimento do QFA}

O QFA possui basicamente dois componentes: uma lista de alimentos, e um espaço para o indivíduo responder com que freqüência consome cada alimento. Quando o objetivo da pesquisa é analisar um ou alguns nutrientes, a lista de alimentos pode ser elaborada a partir da identificação dos alimentos com maior conteúdo do nutriente em questão ${ }^{8,9}$. Se o nutriente de interesse está correlacionado com a energia total consumida, ou se o objetivo é estratificar os indivíduos segundo o seu consumo, a lista de alimentos terá de ser ampliada. Nesse caso, deverá ser constituída pelo maior número possível de alimentos que aportam nutrientes à dieta e também permitir a estimativa de consumo energético ${ }^{10}$. Para elaboração da lista de alimentos podem ser utilizadas diferentes estratégias: a mais simples, porém com maiores limitações, é selecionar os alimentos que aportam os nutrientes de interesse em tabelas de composição de alimentos ou solicitando o auxílio de um especialista em Nutrição. Outra estratégia corresponde à utilização de informações epidemiológicas que verifiquem a existência de associações entre o consumo de um dado fator dietético e a presença de doença. Uma terceira abordagem proposta por Block et al. ${ }^{11}$, considerada a mais apropriada, é a obtenção de uma lista não restrita de alimentos, gerada pela aplicação de vários registros diários ou recordatórios de 24 horas na população. A seguir, é feita uma ponderação estatística, levando-se em consideração a contribuição do alimento para o total consumido, bem como as diferenças interpessoais naquela população.

A freqüência de consumo será registrada em unidades de tempo: dias, semanas, semestres ou anos, podendo contemplar ou não fracionamentos destas unidades. $\mathrm{O}$ formato sugerido é o de perguntas simples e respostas fechadas, com não menos de $5 \mathrm{e}$ não mais que 10 opções, deixando um espaço em branco para aqueles itens de alimentos que ultrapassam o consumo previsto. Este leque de opções produz uma grande e detalhada escala de freqüência, o que é importante, pois os alimentos consumidos menos de uma vez por semana podem ter pouca representatividade no total de nutrientes consumido, porém podem ser importantes para discriminar as categorias de indivíduos ${ }^{12}$.

Na maioria dos estudos observa-se que as dietas se correlacionam de ano para ano e, portanto, a unidade de tempo mais usada para estimar a freqüência de consumo de alimentos é o ano precedente, já que prevê um ciclo completo de estações e as respostas poderiam ser independentes. Outros pontos a serem considerados para se eleger a unidade de tempo são os aspectos fisiológicos da doença estudada e do metabolismo do fator dietético que está sendo analisado.

A inclusão da informação do tamanho de porção consumida dentro do QFA vem sendo um tópico muito discutido, pois os autores verificam que a coleta desta informação não contribui significativamente para melhorar a validade dos QFAs ${ }^{12}$. Existem três formas possíveis de se apresentar os questionários: a primeira é a que prevê a coleta da informação sem a adição do tamanho de porções, ou seja, um questionário simples (qualitativo). A segunda possibilidade é especificar o tamanho de uma porção de referência como parte da pergunta. Assim, por exemplo, a pergunta seria "com que freqüência uma xícara de leite é consumida?", ao invés de se perguntar "quantas vezes o leite é consumido?". Para alimentos que vêm em unidades, como um ovo, uma banana, um pão, esta especificação adicional pode proporcionar clareza à questão. A terceira possibilidade seria se incluir um espaço adicional para cada alimento, no qual o entrevistado descreveria o tamanho de porção usualmente consumida, normalmente com a ajuda de instrumentos visuais.

O papel da memória sempre foi questionado, e esforços para melhorar a qualidade dos questionários têm enfatizado o uso de técnicas cognitivas e elementos para auxiliar a recordação e uma correta estimativa das porções e alimentos consumidos durante $o$ período de interesse ${ }^{13}$. Drewnoski ${ }^{14}$ ressalta 
que, na ausência da memória dos eventos dietéticos, a pessoa confia nas imagens mentais de sua dieta habitual para estimar a quantidade média do alimento questionado. Assim, esta imagem pode incluir as preferências alimentares e é mais uma inferência do que uma recordação.

\section{Estudo de validação}

Nos estudos de validação, o consumo de alimentos ou de nutrientes estimados pelo método escolhido - "test method" - é comparado com outro método de avaliação dietética, julgado mais exato e considerado como padrão de referência.

Neste sentido, o termo "validade" refere-se ao grau com que um instrumento representa bem um objeto medido ${ }^{15}$. Diz-se, então, que uma medição é validada quando está livre de erros sistemáticos ${ }^{12,16}$. Portanto, estudos de validação estimam parâmetros estatísticos desconhecidos dentro de um modelo que especifica relações entre a medição da ingestão e o consumo verdadeiro ${ }^{17}$.

O procedimento de validação descreve a identificação dos erros de medição e não o método do qual se derivam as medidas. A validação, portanto, considera o contexto dentro do qual o método será usado ${ }^{18}$.

Quando se decide validar um instrumento, os propósitos da avaliação dietética e o marco de referência devem estar claramente definidos para posterior identificação dos fatores que poderão confundir o processo de validação.

Validar um QFA exige o questionamento e a definição do período de tempo no qual o processo de validação terá lugar. $\mathrm{O} Q F A$ avalia o consumo usual em um período específico de tempo do passado. Teoricamente, para validar um instrumento é necessária uma medida de referência que avalie o mesmo período relatado. Na prática, os estudos de validação têm mostrado que as estimativas de consumo do QFA são comparadas às médias de consumo calculadas por várias medições da dieta feitas por metodologias como Registros Alimentares, (RA) ${ }^{19-22}$ e Recordatórios de 24horas (R24h) ${ }^{23-26}$, distri- buídas no mesmo período de avaliação do $\mathrm{QFA}^{27}$.

Quando os resultados do questionário são comparados com poucos registros da dieta, a baixa concordância desta comparação pode ser explicada, segundo Nelson ${ }^{18}$, pela variância intrapessoal inerente às avaliações dietéticas de períodos curtos realizados por métodos mais exatos.

\section{Consumo de alimentos vs. consumo de nutrientes}

Para um estudo de validação, são importantes as evidências que a literatura traz a respeito do consumo de alimentos e nutrientes e sua relação com a prevenção ou o risco de algumas doenças ${ }^{28}$. No campo da nutrição, usualmente descreve-se a dieta em termos de conteúdo de nutrientes, mas pode-se também descrevê-la em termos de alimentos e grupos de alimentos, sobretudo na apresentação dos resultados. Ambas as formas de abordagem apresentam vantagens e desvantagens. Segundo Mertz ${ }^{29}$, a proposta científica de se dividir os alimentos em nutrientes e estudar os efeitos sobre a saúde tem sido benéfica. Entretanto, existe o risco de se substituir conhecimentos incompletos de nutrientes por lições aprendidas, fruto de experiências históricas com alimentos. Neste sentido, são necessários cuidados para não se chegar a conclusões erradas.

A relação de causalidade é forte quando se observa o consumo elevado de um determinado nutriente, principalmente quando se tem mais de um alimento fonte e particularmente quando as fontes se apresentam de maneiras diferentes ${ }^{30}$.

Em certo sentido, a associação pode ser confundida pela presença de outros nutrientes ou substâncias. Ou seja, mesmo que tenha sido observada uma associação somente com um alimento fonte, outros fatores contidos em tal alimento poderão ter relações similares com a doença. A hipótese, por exemplo, de que a ingestão de álcool possa causar câncer de mama foi reforçada pela observação não apenas da associação entre ingestão de álcool e câncer de mama, mas também 
por associações independentes com o consumo de cerveja e bebidas destiladas, fazendo com que seja menos provável que algum outro fator, além do álcool nestas bebidas, seja responsável pelo incremento do risco da doença (câncer de mama) ${ }^{31}$.

Deve-se considerar ainda no contexto da validação as inter-relações que derivam dos padrões de comportamento dos indivíduos. Muitas relações recíprocas emergem do uso de alimentos típicos, como por exemplo, os indivíduos que consomem margarina tendem a não comer manteiga, e os usuários de pão integral consomem pouco ou nada de pão branco. Por isto, é recomendável calcular-se a ingestão de nutrientes dos vários grupos de alimentos.

\section{A escolha do método de referência mais adequado}

Por ocasião do desenho de um estudo de validação dois aspectos devem ser considerados: avaliar o quanto o método é bom para se estimar o consumo e a correção dos erros de classificação dos indivíduos de acordo com sua ingestão. Para estimar a exatidão será necessário comparar o método a ser testado (QFA) com outro método (referência) que se julgue superior. Idealmente, $o$ método de referência deverá ser essencialmente diferente e, se possível, conter erros independentes, ou seja, erros não correlacionados com os erros do QFA ${ }^{12,32-4}$.

Sabe-se que a maior fonte de erros relacionados ao QFA são devidos às restrições impostas por uma lista fixa de alimentos, memória, percepção das porções e interpretação das perguntas. Ao se eleger um método de referência, a primeira escolha poderia ser o Registro Alimentar (RA), pois é um método que não depende da memória e os alimentos consumidos são diretamente registrados. Portanto, diminuiriam os erros de percepção e interpretação.

Em situações onde a população é analfabeta, a participação é baixa ou há pouca motivação, múltiplos Recordatórios de $24 \mathrm{~h}$ (R24h) são uma opção razoável. Neste caso, os erros provavelmente estarão parcialmente correlacionados, pois ambos os métodos dependem da memória e da percepção das porções consumidas.

Muitos estudos de validação usam indicadores bioquímicos e isótopos com o intuito de utilizá-los como "padrão ouro" ${ }^{35,36}$. Pufulete et al. ${ }^{22}$, para avaliar a ingestão dietética de ácido fólico, desenvolveram um QFA resumido que foi validado utilizandose medidas de ácido fólico sérico e eritrocitário, e também de registro alimentar de 7 dias por pesadas; Resnicow et al. ${ }^{24}$, com o objetivo de avaliar o consumo de frutas e vegetais, comparou 3 diferentes modelos de QFA, R24h e 3 RA com medidas de carotenóides séricos. Para avaliar o consumo energético de adolescentes, estimados pelo questionário Rockett (YAQ), este instrumento foi validado por Perks et al. ${ }^{37}$ pelo método da água duplamente marcada.

As principais desvantagens são que tais procedimentos mostram-se extremamente caros e avaliam um nutriente de cada vez. Mas, por outro lado, devido ao fato de possuírem erros independentes, garantem e robustecem a precisão do método de referência ${ }^{30}$.

Existem três fontes de erros quando se comparam os resultados da avaliação dietética com parâmetros bioquímicos de referência:

- Diferença entre a avaliação dietética e o consumo verdadeiro;

- Os processos de digestão, absorção, utilização, metabolismo, excreção, mecanismos homeostáticos, podem ter efeitos sobre a relação entre a quantidade ingerida e a medição bioquímica;

- Erros associados ao próprio ensaio bioquímico $^{38}$.

Este tipo de metodologia, em certas circunstâncias, é muito útil, sobretudo quando o método de referência depende de tabelas de composição incompletas. Um exemplo claro é o uso da excreção de sódio urinário para avaliar o consumo de sal dos alimentos.

\section{Seqüência de administração dos métodos}

Os estudos de validação implicam na 
administração de medições repetidas no espaço e períodos de tempo apropriados. Este é um tópico importante durante o delineamento do estudo, pois é possível que a coleta de dados por um método influencie as respostas do outro. Nelson ${ }^{18}$ sugere que o QFA seja administrado antes que o método de referência, por dois motivos: o primeiro é que os indivíduos participantes da pesquisa se encontram independentes de qualquer outra avaliação dietética que poderia influenciar o procedimento de validação. O segundo é que $o$ ato de preencher os formulários do método de referência poderá direcionar a atenção para suas próprias dietas. Por outro lado, se o QFA é preenchido depois do método padrão, os indivíduos poderão modificar suas respostas. Uma forma de solucionar este problema é possibilitar aos participantes da pesquisa a resposta simultânea a ambos os métodos ${ }^{12}$.

\section{Número de pessoas necessárias para um estudo de validação}

A seleção apropriada do número de participantes necessários para um estudo de validação pode ser calculada seguindo a proposta de Willett ${ }^{12}$, que se baseia em uma fórmula padrão $\left({ }^{1} n=(Z \alpha+Z \beta) 2 \sigma^{2} / d^{2}\right)$, na qual se usa transformação de $z$ de Fisher. Documento recente, produto de uma jornada de trabalho, recomenda uma amostra entre $50 \mathrm{e}$ 100 pessoas para cada grupo demográfico ${ }^{39}$.

\section{Número de medições necessárias para um estudo de validação}

Outro aspecto a se considerar é o número de medições necessárias (método referência). Segundo as considerações abordadas anteriormente, o número de dias necessários para se descrever a dieta habitual sobre o período de referência é muito variado. A literatura mostra que podem ser aplicados no mínimo 2, podendo chegar até 28 dias. A primeira situação (poucos dias) pode ser contornada com ajuste estatístico para remover os efeitos da variabilidade intrapessoal $^{12}$.

\section{Outros erros de medição}

Segundo Pérez ${ }^{40}$, os erros de medição podem ocorrer em qualquer fase do estudo:

- Durante o desenho do instrumento;

- Por omissão no protocolo para uso do instrumento (instrumento pouco detalhado);

- Por fraca execução do protocolo durante a coleta dos dados (influência da idade, sexo, raça etc. do indivíduo que responde);

- Por limitações devido às características dos sujeitos (memória fraca para exposição passada);

- Erros durante o processamento e análise dos dados.

Existem também outras formas de erros, de particular interesse, chamados erros diferenciais da medição, que incluem os efeitos biológicos da doença ou do tratamento; os efeitos dos sintomas na fase de pré-diagnóstico da doença; e a consciência do risco da doença.

\section{Medidas de Validade}

A validade da medição da dieta obtida por diversas metodologias é usualmente estudada pela avaliação da concordância em relação ao método de referência. Várias são as propostas existentes que avaliam as medidas de concordância. Dentre elas, as mais usadas e recomendadas são: a comparação de médias (diferenças entre o grupo de médias ou as diferenças entre as medições dentro dos indivíduos), análise de correlação (os coeficientes de correlação de Pearson, Spearman) e a distribuição comparativa por quartis ou quintis da ingestão do nutriente.

\section{Questionários de freqüência alimentar validados no Brasil}

No Brasil existem 4 estudos publicados sobre questionário de freqüência alimentar validado. O primeiro estudo sobre validação dietética foi publicado por Sichieri \& Everhart $^{41}$, onde se fez a validação de um questionário de freqüência alimentar com 
71 itens em comparação com recordatório de 24 horas. Esse questionário foi formulado a partir de dados do ENDEF e de alimentos listados por alguns pesquisadores na área, onde no período de duas semanas aplicaram-se os métodos em 91 funcionários da Universidade Estadual do Rio de Janeiro, para avaliação da dieta no mês anterior.

Cardoso et al. ${ }^{42}$ publicaram estudo avaliando a validade e reprodutibilidade de um questionário de freqüência alimentar com 120 itens, aplicado em mulheres nipo-brasileiras, em comparação com 12 registros alimentares por pesagem de alimentos. Em relação à validação do questionário, no período de um ano, 55 mulheres foram acompanhadas.

Slater et al. ${ }^{43}$ desenvolveram e validaram um questionário semiquantitativo de freqüência alimentar com 76 itens para adolescentes comparando-o com 3 recordatórios de 24 horas, no período de 6 meses. Os resultados do estudo revelaram uma alta variabilidade no consumo da dieta dos adolescentes, mostrando razões de variância extremamente altas para o colesterol, retinol e vitamina C.

Por fim, Salvo \& Gimeno $^{44}$ avaliaram a reprodutibilidade e a validade de um questionário de freqüência alimentar com 97 itens alimentares para a população adulta com excesso de peso, utilizando como referência o recordatório 24 horas, aplicado três vezes com intervalo de 15 dias.

\section{Considerações finais}

A seleção ou desenvolvimento de um instrumento de medição é obviamente um passo critico no delineamento de um estudo epidemiológico. Não podendo esquecer que a primeira atitude a ser tomada deverá ser revisar a literatura disponível sobre estudos de validação de instrumentos que avaliem o fator dietético que pretende-se estudar e também a relação deste com a doença. No caso de inexistência ou de substanciais diferenças será necessário avaliar a confiabilidade e, de preferência, a validade do novo instrumento a ser testado.

Estudos de validação de QFA procuram quantificar os erros de medição do método. Destaca-se que erro de medição não é uma propriedade inerente a um instrumento e sim uma propriedade de um instrumento aplicado de uma maneira particular em uma população especifica. Por tal razão, o erro pode variar não somente entre dois instrumentos que medem a mesma exposição, mas também em um único instrumento quando aplicado de forma diferente ou aplicado em diferentes grupos populacionais.

\section{Referências}

1. Sampson L. Food frequency questionnaires as a research instrument. Clin Nutr 1985; 4: 171-8.

2. Ocké MC, Bueno-de-Mesquita HB, Goddijn HE, Jansen A et al. The Dutch EPIC food frequency questionaire. I. Description of the questionnaire, and relative validity and reproducibility for food groups. Intern J Epidemiol 1997; 26 Suppl 1: 37S-48S.

3. Willett WC. Future directions in the development of food-frequency questionnaires. Am J Clin Nutr 1994; 59 Suppl: 171S-4S.

4. Abramson JH, Slome C, Kosovsky C. Food frequency interview as an epidemiological tool. Am J Pub Health 1963; 53: 1093.
5. Gibson RS. Principles of Nutritional Assessment. Oxford: Oxford University Press; 1990.

6. Armstrong BK, White E, Saracci R. Principles of exposure measurement in epidemiology. $2^{\text {nd }}$ ed. Oxford: Oxford University Press; 1995.

7. Jimenez LG, Martín-Moreno JM. Cuestionario de frecuencia de consumo alimentario. In: Majem LIS, Aranceta BJ, Verdú MJ. Nutricíon y Salud Publica: métodos, bases científicas e aplicaciones. España: Masson; 1995. p. 120-5.

8. Nelson M, Hague GF, Cooper C, Bunkers VW. Calcium Intake in the elderly: validation of a dietary questionnaire. J Human Nutr Diet 1989; 1: 115-27. 
9. Pufulete M, Emery PW, Nelson M, Sanders TA. Validation of a short food frequency questionnaire to assess folate intake. Br J Nutr 2002; 87(4): 383-90.

10. Zulkifli SN, Yu SM. The food frequency method for dietary assessment. J Am Diet Assoc 1992; 92: 681-5.

11. Block G, Hartman AM, Dresser CM, Carroll MD, Gannon J, Gardner L. A data-basedapproach to diet questionnaire design and testing.Am J Epidemiol. 1986;124(3):453-69.

12. Willet WC. Nutritional Epidemiology. $2^{\text {nd }}$ ed. Oxford: Oxford University Press; 1998.

13. Subar AF, Thompson FE, Smith AF, Jobe JB et al. Improving food frequency questionnaire: a qualitative aproach using cognitive interviewing. J Am Diet Assoc 1995; 95: 781-8.

14. Drewnowoski A. Diet image: a new perspective on the Food-Frequency Questionnaire. Nutr Rev 2001; 59: 370-4.

15. Pereira MG. Epidemiologia: teoria e prática. Rio de Janeiro: Guanabara Koogan; 2001.

16. Vioque LJ. Validez de la evaluacioón de la ingesta alimentar. Nutrición y Salud Pública - Métodos, bases científicas y aplicaciones. España: Masson, S.A.; 1995.

17. Kaaks R, Riboli E, Estève J, van Kappel AL, van Staveren WA. Estimating the accuracy of dietary questionnaire assessments: validation in terms of structural equation models. Stat Med 1994; 13: 127-42.

18. Nelson M. The validation of dietary assessment. In: Margetts B, Nelson M. Design concepts in nutrition epidemiology. $2^{\text {nd }}$ ed. Oxford: Oxford University Express; 1997. p. 241-72.

19. Persson LA, Cargren G. Measuring children's diets: evaluation of dietary assessmnent techniques in infancy and childhood. Int J Epidemiol 1984; 13: 506-17.

20. Ianetti RJ, Zuckerman AE, Blyer EM, O'Vrien et al. Comparison of dietary intake methods with young children. Psychol Rep 1994; 74: 883-9.

21. Taylor RW, Goulding A. Validation of a short food frequency questionnaire to assess calcium intake in children aged 3 to 6 years. Eur J Clin Nutr 1998; 52: 46465.

22. Pufulete M., Emery PW. Nelson M, Sanders TAB. Validation of a short food frequency questionnaire to assess folate intake. Br J Nutr 2002; 87: 383-90.

23. Blum RE, Wei EK, Rockett HR, Langeliers JD, Leppert J, Gardner JD, Colditz GA. Validation of a food frequency questionnaire in Native American and Caucasian children 1 to 5 years of age. Matern Child Health J. 1999; 3(3):167-72.
24. Resnicow K, Odom E, Wang T, Dudley WN et al. Validation of three food frequency questionnaires and 24-hour recalls with serum carotenoid levels in a sample of African-American adults. Am J Epidemiol 2000; 152(11): 1072-80.

25. Rodriguez MM, Mendez H, Torun B, Schroeder D, Stein AD. Validation of a semi-quantitative food-frequency questionnaire for use among adults in Guatemala. Public Health Nutr 2002; 5(5):691-9.

26. Parrish LA, Marshall JA, Krebs NF, Rewers M et al. Validation of a food frequency questionnaire in preschool children. Epidemiology 2003, 14(2): 213-7.

27. Lopez VJ. Validez de la evaluación de la ingesta dietética. In: Serra Majem Ll. Nutrición y Salud Pública. Metodos, bases científicas y aplicaciones. España: Ed. Masson; 1995. p. 132-40.

28. Peto R, Doll R, Buckley JD, Sporn MB. Can dietary betacarotene materially reduce human cancer rates? Nature 1981; 290: 201-8.

29. Mertz W. Foods and nutrients. J Am Diet Assoc 1984; 84: $769-70$.

30. Willett WC. Diet and nutrition. In: Schottenfeld D, Fraumeni JF. Cancer epidemiology and prevention. $2^{\text {nd }}$ ed. Oxford: Oxford University Press; 1996. p. 438-61.

31. Willett WC. Nutritional Epidemiology. In: Rothman KJ, Greenland S. Modern Epidemiology. Lippincot (USA): Williams \& Wilkins; 1998. p. 623-42.

32. BGA Commission on Nutritional Epidemiology. Recommendations for the design and analysis of nutritional epidemiologic studies with measurement errors in the exposure variables. Eur J Clin Nutr 1993; 47 Suppl 2: 53S-57S.

33. Day NE, Ferrari P. Some methodological issues in nutritional epidemiology. IARC Sci Publ 2002; 156: 5-10.

34. Day NE, Mckeown N, Wong MY, Welch A, Bingham S. Epidemiological assesment of diet: a comparison of a 7-day diary with a food frquency questionnaire using urinary markers of nitrogen, potassium and sodium. Int J Epidmiol 2001, 30: 309-17.

35. Black AE, Prentice AM, Goldberg GR, Jebb SA, Bingham SA, Living-stone MBE et al., Measurements of total energy expenditure provide insights into the validity of dietary measurement of energy intake. J Am Diet Assoc 1993; 93: 572-9.

36. Prentice AM, Coward WA, Davies HL, Murgatroyd PR et al. Unexpectedly low levels of energy expenditure in healthy women. Lancet 1985; i: 1419-22.

37. Perks SM, Roemmich JN, Sandow-Pajewski M, Clark PA, Thomas E, Weltman A, Patrie J, Rogol AD. Alterations in growth and body composition during puberty. IV. Energy intake estimated by the youthadolescent food-frequency questionnaire: validation by the doubly labeled water method. Am J Clin Nutr 2000; 72(6): 1455-60. 
38. Hunter DJ. Biochemical indicators of dietary intake. In: Willett W. Nutritional epidemiology. $2^{\text {nd }}$ ed. Oxford: Oxford University Press; 1998. p. 174-243.

39. Burley V, Cade J. Consensus document on the development, validation and utilization of food frequency questionnaires. [The Fourth International Conference on Dietary Assessment Methods; 2000 sept 17-20; Tucson, Arizona (USA)].

40. Perez RC. Fuentes de error en la evaluación del consumo de alimentos. In: Majem SLl, Aranceta BJ, Verdú MJ. Nutrición y Salud Pública - Métodos, bases científicas y aplicaciones. España: Masson, S.A.; 1995. p.168-72.

41. Sichieri R. Epidemiologia da obesidade. Rio de Janeiro: Ed. UERJ, 1998.
42. Cardoso MA, Stocco PR Desenvolvimento de um questionário quantitativo de freqüência alimentar em imigrantes japoneses e seus descendentes residentes em São Paulo, Brasil. Cad Saúde Pública 2000; 16 (1):107-114.

43. Slater B, Philippi ST, Fisberg RM, Latorre MR. Validation of a semi-quantitative adolescent food frequency questionnaire applied at a public school in Sao Paulo, Brazil. Eur J Clin Nutr 2003; 57(5):629-35.

44. Salvo VL, Gimeno SG. [Reproducibility and validity of a food frequency questionnaire]. Rev Saude Publica 2002; 36(4):505-12.

Recebido em: 02/07/2002 Versão final reapresentada em: 02/04/2003 Aprovação em: 14/04/2003 